\title{
Die Digitalisierung aus Ärztesicht (Teil II)
}

\author{
Fabian Röthlisberger ${ }^{a}$, Reinhold Sojer ${ }^{b}$, Tarja Zingg ${ }^{c}$, Oliver Raykid \\ ${ }^{a}$ Wissenschaftlicher Mitarbeiter Digitalisierung/eHealth, FMH; ${ }^{b}$ Dr. rer. biol. hum., Leiter Digitalisierung/eHealth, FMH; ${ }^{c}$ Dr. oec. publ. \& BA of Arts
} in Design, Geschäftsleitung Lumina Health; ${ }^{d}$ Dipl.-Psych., selbständiger wissenschaftlicher Berater Statistik und Datenmanagement

FMH und KPMG untersuchten in einer gemeinsamen Studie die Haltung der Schweizer Ärzteschaft in Bezug auf die Digitalisierung und die Nutzung digitaler Gesundheitsangebote [1]. Im ersten Teil der publizierten Studie konnte gezeigt werden, dass die Ärzteschaft grundsätzlich positiv gegenüber digitalen Gesundheitsangeboten eingestellt ist. Ärztinnen und Ärzte, welche digitale Gesundheitsangebote im beruflichen Alltag integrieren, nutzen insbesondere digitale Angebote in den Bereichen Administration, Kommunikation und Information sowie im Bereich der Therapieunterstützung. Dennoch werden digitale Angebote, aufgrund fehlender Anreize und fehlender Standards für die integrative Datennutzung, häufig noch nicht in der Praxis integriert. Auch steht die Mehrheit der Befragten der Telekonsultation kritisch gegenüber. Eine Limitation der Studie war, dass einige der Ergebnisse stark korrelieren und dass Durchschnittswerte die komplexe Realität nicht genügend widerspiegeln [2]. Aus diesem Grund wurde in einem zweiten Schritt eine Clusteranalyse durchgeführt, um homogene Gruppen von Ärztinnen und Ärzten zu identifizieren, welche sich in ihrem Antwortverhalten gleichen.

\section{Methoden}

Mit dem Ziel, das Antwortverhalten der Ärzteschaft möglichst nicht hypothesengeleitet zu typologisieren, wurde anhand der erhobenen Daten eine hierarchische Clusteranalyse (Agglomerationsmethode nach Ward) durchgeführt. In einem zweiten Schritt wurde das Verfahren der Diskriminanzanalyse eingesetzt, um die Stabilität der Trennbarkeit der Subgruppen zu verifizieren. Bei der Diskriminanzanalyse erfolgt eine Prädiktion der durch die Clusteranalyse identifizierten Gruppen. Abschliessend wurde anhand einer Kreuzvalidierung die Stabilität dieser Prädiktion getestet. ${ }^{1}$

\footnotetext{
1 Die Trefferquote aus der Kreuzvalidierung lag bei $73 \%$, was für ein sehr gutes Ergebnis spricht.
}

unterscheiden und unterschiedliche soziodemographische Variablen aufweisen (Abb. 1).

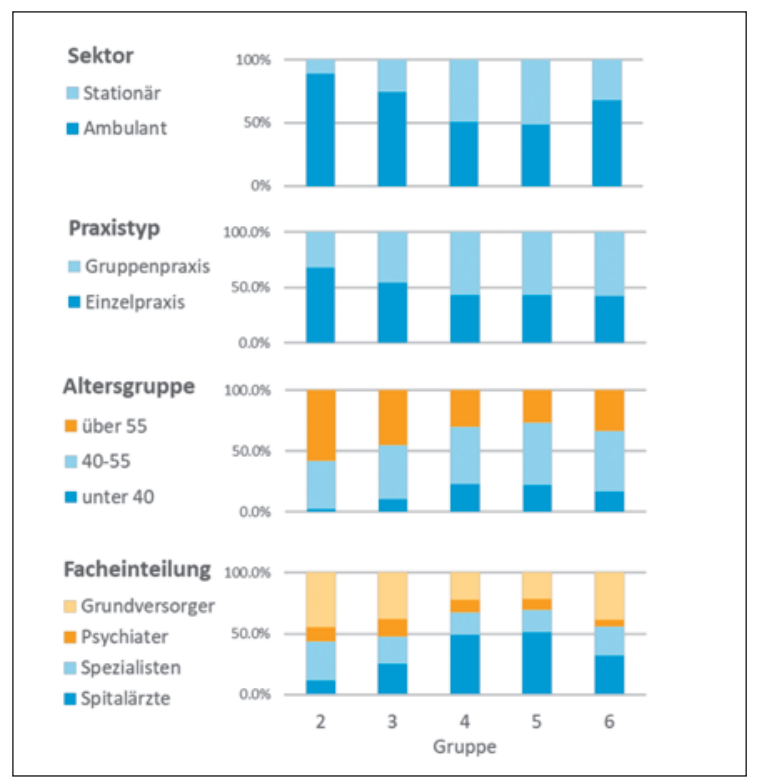

Abbildung 1: Soziodemographische Variablen der Clustergruppen (ohne Gruppe 1).

Gruppe $1(n=438,9,6 \%)$ zeichnet sich durch eine hohe Anzahl an fehlenden Daten aus, weshalb das Antwortverhalten dieser Gruppe im Folgenden nicht weiter beschrieben wird. Die Identifikation einer Gruppe mit vielen fehlenden Daten ermöglicht jedoch präzisere Aussagen in den übrigen Gruppen.

Gruppe 2 ( $n=202,4,4 \%$ ) und Gruppe 3 ( $n=1346,29,5 \%)$ beinhalten eine Ärzteschaft, welche häufig ein höheres Alter aufweist und oft ambulant sowie als Grundversorger tätig ist. Gruppe 2 beinhaltet dabei im Gruppenvergleich den grössten Anteil an Ärztinnen und Ärzten aus Zürich (25\%).

Gruppe 4 ( $n=1116,24,4 \%)$ und Gruppe 5 ( $n=514,11,2 \%)$ zeichnen sich durch eine jüngere Ärzteschaft sowie einen hohen Anteil an stationär tätigen Ärztinnen und Ärzten aus. Gruppe 5 zeichnet sich zudem durch einen hohen Anteil an Ärztinnen und Ärzten aus der Genferseeregion aus (24\%). 
Gruppe 6 ( $\mathrm{n}=954,20,9 \%)$ beinhaltet im Vergleich zu Gruppen 4 und 5 eine vermehrt ambulant tätige Ärzteschaft und häufiger Grundversorger. Gegenüber Gruppen 2 und 3 zeichnet sich Gruppe 6 durch einen höheren Anteil der in Gruppenpraxen tätigen Ärzteschaft sowie einen höheren Anteil von Spitalärzten aus.

\section{Generelle Einstellung und Integration von digitalen Angeboten}

Gruppen 2 und 3 zeichnen sich im Vergleich zu den Gruppen 4-6 durch eine skeptische Einstellung gegenüber digitalen Gesundheitsangeboten aus. ${ }^{2}$ Beide Gruppen integrieren nur selten digitale Angebote in ihrem Praxisalltag (Gruppe 2: 3\%, Gruppe 3: 11\%) und geben an, nur selten von Patientinnen und Patienten darauf angesprochen zu werden. Die Qualität der Daten, welche über digitale Gesundheitsangebote bereitgestellt wird, bewerten in Gruppe 2 nur 4\% eher oder sehr positiv, und die Nutzung von digitalen Angeboten wird sowohl in Gruppe 2 wie 3 von weniger als 15\% der Ärzteschaft gelegentlich oder oft empfohlen.

Gruppen 4-6 zeichnen sich durch wenig Skepsis gegenüber digitalen Gesundheitsangeboten aus, wobei $99 \%$ der Gruppe 5 auf die Frage nach der Einstellung gegenüber digitalen Angeboten eher positiv oder sehr positiv antworten. Die Befragten der Gruppe 5 empfehlen digitale Gesundheitsangebote gegenüber Gruppen 4 und 6 häufiger ihren Patienten (73\%) und erwarten häufiger eine Veränderung ihrer Aufgaben (72\%). Fast drei Viertel der Befragten dieser Gruppe integrieren digitale Angebote in ihren Praxisalltag (72\%). ${ }^{3}$ Gruppe 4 zeichnet sich im Vergleich zu Gruppe 6 mit einer Ärzteschaft aus, die zu 98\% angibt, selten oder nie von ihren Patienten auf digitale Gesundheitsangebote angesprochen zu werden. Weiter integrieren die Befragten aus Gruppe 4 digitale Angebote weniger häufig "gelegentlich oder oft» in ihren Praxisalltag als Gruppe 6 (9 vs. 71\%).

\section{Nutzen}

Der Nutzen digitaler Gesundheitsangebote für administrative Unterstützung, Telekonsultation, Verhaltensänderung oder Patienteninformation wird von über 90\% der Befragten aus Gruppe 2 skeptisch oder sehr skeptisch beurteilt, wohingegen die Hälfte der Ärztinnen und Ärzte aus Gruppe 3 den Nutzen von digitalen Angeboten eher positiv oder sehr positiv bewertet (Ausnahme Telekonsultation: 15\%).

Die Gruppen 4-6 bewerten den Nutzen von digitalen Gesundheitsangeboten positiver als Gruppen 2 und 3. Einen Nutzen für administrative Unterstützung, Verhaltensänderung und Patienteninformation wird von den Befragten der Gruppe 5 von über 95\% eher positiv oder sehr positiv beurteilt. Im Vergleich zu Gruppen 4 und 6 beurteilen die Ärztinnen und Ärzte der Gruppe 5 den Nutzen von Telekonsultationen signifikant positiver (85 vs. 32\%).

\section{Bedenken und Hürden}

Die Fragen nach Bedenken gegenüber digitalen Gesundheitsangeboten werden von Gruppe 2 am häufigsten mit «ja» beantwortet. $82 \%$ befürchten, dass der Umgang mit Informationen zu Fehlinterpretationen führen kann. 72\% teilen die Bedenken, dass der Patient den Arzt aufgrund der digitalen Angebote zu häufig oder zu selten aufsucht. $68 \%$ antworten, dass die digitalen Angebote mehrheitlich keine validen Informationen bieten. In allen Gruppen werden Bedenken zum Datenschutz (21-33\%) oder zu einem grösser werdenden Arbeitsaufwand (2-17\%) von einem kleinen Anteil an Ärztinnen und Ärzten geäussert. ${ }^{4}$

In Bezug auf den Einsatz von digitalen Angeboten in ihrer Gesundheitseinrichtung bewertet Gruppe 2 fehlende technische Möglichkeiten häufiger als Hürde (35\%) als die anderen Gruppen (12-21\%). Weiter stellt die ungenügende Abbildung von digitalen Angeboten in den Tarifen für Gruppen 2 und 3 eine grössere Hürde als für Gruppen 4-6 dar. Umgekehrt bewerten circa zwei Drittel der Gruppen 4-6 die politischen Rahmenbedingungen als Hürde, während bei Gruppen 2 und 3 diese Meinung von etwas mehr als der Hälfte der Befragten geteilt wird. Fehlende Standards und die Interoperabilität mit den eigenen Systemen werden in allen Gruppen von einer Mehrheit der Befragten als Hürde für den Einsatz von digitalen Angeboten angesehen (56-65\%).

\section{Anreiz zu digitalisieren}

Auf die Frage nach dem Anreiz, ihre Gesundheitseinrichtung zu digitalisieren, antworten die Ärztinnen und Ärzte aus Gruppe 2 am häufigsten, dass der Mehrnutzen für den Patienten für sie ein Anreiz ist (46\%). Für die Befragten der Gruppe 2 (31\%) und Gruppe 3 (62\%) stellt der Wettbewerbsdruck im Vergleich zu den Gruppen 4 bis 6 (über 70\%) seltener einen Anreiz dar, um ihre Gesundheitseinrichtung zu digitalisieren. Weiter gibt die Ärzteschaft aus Gruppe 5 im Vergleich zu den Befragten der Gruppen 4 und 6 häufiger zur Antwort, dass eine angemessene Tarifierung (22\%) und gesetzliche Verpflichtungen (68\%) für sie einen Anreiz zur Digitalisierung darstellen.

\section{Diskussion}

Im Gruppenvergleich fällt auf, dass die Gruppen 2 und 3 mit einem grossen Anteil an älteren und ambulant tätigen Grundversorgern sowie Spezialisten grosse Vorbehalte gegenüber digitalen Gesundheitsangebo- 


\begin{tabular}{|c|c|c|c|c|c|}
\hline & Gruppe 2 & Gruppe 3 & Gruppe 4 & Gruppe 5 & Gruppe 6 \\
\hline Verschiedene Fragen & \multicolumn{5}{|c|}{ eher positiv / sehr positiv oder gelegentlich / oft } \\
\hline Wie ist prinzipiell ihre Einstellung gegenüber digitalen Gesundheitsangeboten? & $2 \%$ & $29 \%$ & $76 \%$ & $99 \%$ & $77 \%$ \\
\hline Wurden sie bereits aktiv auf digitale Gesundheitsangebote angesprochen? & $9 \%$ & $2 \%$ & $2 \%$ & $40 \%$ & $43 \%$ \\
\hline Integrieren Sie digitale Gesundheitsangebote in ihrem Praxisalltag? & $3 \%$ & $11 \%$ & $9 \%$ & $72 \%$ & $71 \%$ \\
\hline Wie schätzen Sie die Qualităt der Daten ein, die bereitgestellt werden? & $4 \%$ & $15 \%$ & $29 \%$ & $51 \%$ & $34 \%$ \\
\hline $\begin{array}{l}\text { Empfehlen Sie Ihren Patienten auch ohne Nachfrage die Nutzung von digit } \\
\text { Gesundheitsangeboten? }\end{array}$ & $2 \%$ & $13 \%$ & $20 \%$ & $73 \%$ & $54 \%$ \\
\hline $\begin{array}{l}\text { Erwarten Sie eine Veränderung ihrer Aufgaben durch die Nutzung von digitalen } \\
\text { Gesundheitsangeboten? }\end{array}$ & $48 \%$ & $45 \%$ & $53 \%$ & $72 \%$ & $67 \%$ \\
\hline Nutzen & \multicolumn{5}{|c|}{ eher positiv / sehr positiv } \\
\hline Administrative Unterstützung (onlineTerminvereibarung, Rezeptanforderung) & $7 \%$ & $52 \%$ & $91 \%$ & $97 \%$ & $74 \%$ \\
\hline Onlinesprechstunde und Telekonsultationen (Telemedizin) & $0 \%$ & $15 \%$ & $32 \%$ & $85 \%$ & $32 \%$ \\
\hline Digitale Unterstützung zur Verhaltensänderung & $7 \%$ & $47 \%$ & $87 \%$ & $95 \%$ & $81 \%$ \\
\hline Digitale Patienten Informationen über die eigene Erkrankung & $6 \%$ & $47 \%$ & $87 \%$ & $98 \%$ & $83 \%$ \\
\hline Bedenken & \multicolumn{5}{|c|}{ Ja-Antworten } \\
\hline Umgang mit den Informationen kann zu Fehlinterpretationen führen & $82 \%$ & $70 \%$ & $61 \%$ & $51 \%$ & $61 \%$ \\
\hline Patient kann zu falschen Selbstbehandlungen animiert werden & $74 \%$ & $63 \%$ & $54 \%$ & $37 \%$ & $53 \%$ \\
\hline Patient eignet sich zu viel Pseudowissen an & $54 \%$ & $37 \%$ & $24 \%$ & $19 \%$ & $33 \%$ \\
\hline Patient sucht den Arzt dadurch zu selten oder zu häufig auf & $72 \%$ & $52 \%$ & $46 \%$ & $46 \%$ & $52 \%$ \\
\hline Datenschutz ist nicht genügend gewährleistet & $33 \%$ & $25 \%$ & $26 \%$ & $21 \%$ & $26 \%$ \\
\hline Zuverlässigkeit der Geräte ist ungenügend & $51 \%$ & $27 \%$ & $19 \%$ & $15 \%$ & $20 \%$ \\
\hline Die Angebote bieten mehrheitlich keine validen Informationen & $68 \%$ & $55 \%$ & $37 \%$ & $26 \%$ & $46 \%$ \\
\hline Der Arbeitsaufwand wird grōsser & $17 \%$ & $7 \%$ & $2 \%$ & $7 \%$ & $9 \%$ \\
\hline Hürden & \multicolumn{5}{|c|}{ Ja-Antworten } \\
\hline fehlender Nutzen-Nachweis & $43 \%$ & $32 \%$ & $28 \%$ & $37 \%$ & $37 \%$ \\
\hline politische Rahmenbedingungen & $53 \%$ & $55 \%$ & $67 \%$ & $63 \%$ & $64 \%$ \\
\hline fehlende Standards, Interoperabilität mit eigenen Systemen & $64 \%$ & $59 \%$ & $56 \%$ & $62 \%$ & $65 \%$ \\
\hline Keine Abgeltung, ungenügende Abbildung in den Tarifen & $53 \%$ & $49 \%$ & $38 \%$ & $30 \%$ & $43 \%$ \\
\hline Zeitmangel & $17 \%$ & $22 \%$ & $23 \%$ & $25 \%$ & $18 \%$ \\
\hline fehlende technische Möglichkeiten & $35 \%$ & $18 \%$ & $12 \%$ & $12 \%$ & $21 \%$ \\
\hline Anreiz zu digitalisieren & \multicolumn{5}{|c|}{ Ja-Antworten } \\
\hline gesetzliche Verpflichtung & $38 \%$ & $54 \%$ & $62 \%$ & $68 \%$ & $66 \%$ \\
\hline angemessene Tarifierung & $2 \%$ & $8 \%$ & $15 \%$ & $22 \%$ & $14 \%$ \\
\hline Wettbewerbsdruck gegenüber digitalisierten Praxen & $31 \%$ & $62 \%$ & $77 \%$ & $76 \%$ & $71 \%$ \\
\hline mehr Nutzen für den Patienten & $46 \%$ & $15 \%$ & $6 \%$ & $12 \%$ & $19 \%$ \\
\hline
\end{tabular}

Abbildung 2: Antwortverhalten nach Clustergruppen (ohne Gruppe 1).

ten haben. Die Ergebnisse des eHealth-Barometers 2018 zeigen ähnliche Ergebnisse in Bezug auf die Einstellung zum elektronische Patientendossier: 71\% der Spitalärzte und nur 53\% der Praxisärzte beurteilen den Wert des elektronischen Patientendossiers positiv [3]. Die Clusteranalyse zeigt allerdings auch, dass die ambulant tätige Ärzteschaft nicht per se mit einer Skepsis gegenüber digitalen Gesundheitsangeboten behaftet ist, denn drei Viertel der Gruppe 6, die sich durch einen grossen Anteil an jungen Ärztinnen und Ärzten auszeichnet, sind positiv eingestellt.

Dass jüngere Ärztinnen und Ärzte gegenüber digitalen Anwendungen eine positivere Einstellung als ihre älteren Kolleginnen und Kollegen haben, wurde auch in einer amerikanischen Studie zur Erhebung der Digitalisierung innerhalb der ambulant tätigen Ärzteschaft in 
5 Status quo der IT-Nutzung in Schweizer Arztpraxen.

62017 wurde das «Meaningful Use»-Programm im neuen «Merit-based Incentive Payment System» integriert.
Die Literatur zu diesem Artikel findet sich online unter www.saez.ch $\rightarrow$ aktuelle Ausgabe oder $\rightarrow$ Archiv $\rightarrow$ Ausgabe 48 .
Korrespondenz: Dr. Reinhold Sojer Leiter Abteilung

Digitalisierung/eHealth

FMH

Elfenstrasse 18

Postfach 300

CH-3000 Bern 15

Tel. 0313591204

reinhold.sojer[at]fmh.ch den Jahren 2009 bis 2013 festgestellt [4]. Allerdings zeigt die Schweizer SISA-Studie ${ }^{5}$ mit 685 befragten ambulant tätigen Ärztinnen und Ärzten, dass die positive Einstellung nicht nur durch das Alter erklärt werden kann. Vielmehr beeinflussen Faktoren wie Fachrichtung oder Praxisgrösse die Bereitschaft zur Digitalisierung der eigenen Praxisprozesse [5]. Dies könnte auch den höheren Anteil an Gruppenpraxen in den positiv eingestellten Gruppen 4-6 erklären.

\section{Mehrnutzen für die Patientinnen und Patienten}

Die Analyse der Gruppe mit den stärksten Bedenken (Gruppe 2) zeigt, dass die Mehrheit den Nutzen ihnen bekannter digitaler Angebote in Frage stellt. Es erstaunt daher nicht, dass sie ihre Gesundheitseinrichtung erst dann digitalisieren wollen, wenn der Mehrnutzen für die Patienten aus ihrer Sicht zunimmt. Zudem beurteilen die Ärztinnen und Ärzte mit Praxistätigkeit die fehlende Interoperabilität und mangelnden technischen Möglichkeiten sowie die ungenügende tarifliche Abbildung als Hemmnisse bei der digitalen Integration.

\section{Investitionskosten bei ungenügender tariflicher Abbildung}

Gerade für Einzelpraxen stellen die hohen Investitionskosten bei ungenügender tariflicher Abbildung eine grosse Hürde für die Integration von digitalen Gesundheitsangeboten dar [6]. Ein Bericht des Commonwealth Fund gibt jedoch zu bedenken, dass finanzielle Anreize Ärztinnen und Ärzte mit grössten Bedenken nicht umzustimmen vermögen, und empfiehlt weitere Massnahmen: Digitale Angebote mit klinischen Entscheidungshilfen oder Ausgleichszahlungen für Ärztinnen und Ärzte, die sich aktiv an einem Datenaustausch beteiligen, können Anreize schaffen, um digitale Angebote vermehrt in den Praxisalltag zu integrieren [7]. In den USA wird seit mehreren Jahren ein unter dem Namen «Meaningful Use» bekanntes Anreizsystem eingesetzt, bei dem die Ärzteschaft für qualitätssteigernde Aktivitäten im Bereich der Digitalisierung finanziell belohnt wird ${ }^{6}[8,9]$. Jedoch glaubt nur $1 / 5$ der amerikanischen Ärzteschaft an eine Verbesserung der Behandlungsqualität durch diese Initiative. Dies ist auf eine Unzufriedenheit mit dem elektronischen Patientendossier zurückzuführen, weswegen der Einbezug der Ärzteschaft in der Umsetzung solcher nationalen Programme als Erfolgsfaktor angesehen wird $[10,11]$.

\section{Barrieren zur Nutzung von Telemedizin}

Im ersten Teil der publizierten Ergebnisse wurde beschrieben, dass Ärztinnen und Ärzte den Nutzen von Telekonsultationen mehrheitlich negativ einschätzen. Eine Metaanalyse aus dem Jahr 2018 stellt verschie- dene Gründe für eine solche Einschätzung dar: technisch überfordertes Personal, Widerstand gegen Veränderungen, ungenügende finanzielle Anreize und das unterschiedliche Alter oder der Bildungsstand der Patienten [12]. In einer anderen Studie wird der Verlust des persönlichen Kontaktes sowie die Einstellung der Patienten als weiteres Hemmnis für den Einsatz von Telekonsultationen beschrieben [13]. Anhand der Clusteranalyse konnte jedoch gezeigt werden, dass die Gruppe von jüngeren und häufig stationär tätigen Ärztinnen und Ärzten den Nutzen von Telekonsultationen durchaus sehr positiv bewertet. Das Verständnis für Beweggründe der unterschiedlichen Bewertung von Telekonsultationen bedarf weiterer Analysen

Betrachtet man die Nachfrageseite, also die Patientinnen und Patienten, sieht die Bilanz allerdings ganz anders aus: Eine grosse Mehrheit möchte zunehmend mit ihren Ärztinnen und Ärzten via E-Mail, Messenger, Telefon oder per Video digital kommunizieren, ohne dabei eine Praxis aufsuchen zu müssen. Dies belegen verschiedene Studien statistisch repräsentativer Umfragen $[3,14]$ sowie beispielsweise die über 6 Millionen Telekonsultationen, welche seit dem Jahr 2000 durch das Medgate Telemedicine Center durchgeführt wurden [15].

\section{Zusammenfassung und Ausblick}

Die Studie zeigt, dass die Ärzteschaft gegenüber digitalen Angeboten grundsätzlich positiv eingestellt ist, es aber Untergruppen von häufig älteren und ambulant tätigen Ärztinnen und Ärzten gibt, die mehr Skepsis zeigen. Um diese Gruppen zur Digitalisierung im Dienst der Patienten zu motivieren, müssen einerseits Hürden wie die fehlende Interoperabilität, fehlende technische Möglichkeiten sowie die fehlende tarifliche Abbildung reduziert werden. Andererseits müssen Bedenken ernst genommen und der Nutzen von digitalen Angeboten für diese Ärzteschaft noch stärker ersichtlich werden. Bei der sehr positiv eingestellten Ärzteschaft konnte eine Gruppe mit einem hohen Anteil an jüngeren und stationär tätigen Ärztinnen und Ärzten identifiziert werden, welche im Gegensatz zu den Ergebnissen aus dem ersten Teil der Studie gerade die digitale Kommunikation mit den Patientinnen und Patienten sehr positiv bewerten Weitere Analysen in dieser Subgruppe könnten interessant sein, um mehr über die positiven Erfahrungen mit Telekonsultationen zu erfahren. In der Studie kommt auch zum Ausdruck, dass sich Nutzen und Aufwand der Digitalisierung je nach Haltung, Grösse der Organisationseinheit und des Anwendungsbereichs unterscheiden 


\section{Literatur}

1 Giger MA, Nörenberg N. Digitalisierung im Schweizer

Gesundheitswesen: Das neue Rollenverständnis der Schweizer

Ärztinnen und Ärzte. Clarity Healthc. 2018; pp. 14-21.

2 Sojer R, Röthlisberger F, Rayki O. Angebot und Nachfrage von digitalen Gesundheitsangeboten (Teil I). Schweiz Ärzteztg. 2018;99(42): 1428-31.

3 Golder L and Jans C. Swiss eHealth Barometer 2018, 2018.

4 Wylie MC, Baier RR, Gardner RL. Perceptions of Electronic Health Record Implementation: A Statewide Survey of Physicians in Rhode Island. Am J Med. 2014 Oct 2014;127(10):1010.e21-7.

5 Djalali S, Ursprung N, Rosemann T, Senn O, Tandjung R. Undirected health IT implementation in ambulatory care favors paper-based workarounds and limits health data exchange. Int J Med Inform. 2015.

6 Ross J, Stevenson F, Lau R, Murray E. Factors that influence the im plementation of e-health: A systematic review of systematic reviews (an update). Implementation Science. 2016.

7 Blumenthal D. The Federal Role in Promoting Health Information Technology. 2009.

8 Medicare Program; Merit-Based Incentive Payment System (MIPS) and Alternative Payment Model (APM) Incentive Under the Physician Fee Schedule, and Criteria for Physician-Focused Payment Models. Final rule with comment period. Fed Regist. 2016.

9 Doherty RB. Goodbye, sustainable growth rate-hello, merit-based incentive payment system. Ann Intern Med. 2015.

10 Emani S, Ting DY, Healey M, Lipsitz SR, Karson AS, Bates DW. Physician Beliefs about the Meaningful Use of the Electronic Health Record: A Follow-Up Study. Appl Clin Inform. 2017.

11 Makam AN, et al. Use and satisfaction with key functions of a common commercial electronic health record: A survey of primary care providers. BMC Med Inform Decis Mak. 2013.

12 Scott Kruse C, Karem P, Shifflett K, Vegi L, Ravi K, Brooks M. Evaluating barriers to adopting telemedicine worldwide: A systematic review. Journal of Telemedicine and Telecare. 2018.

13 Kayyali R, Hesso I, Mahdi A, Hamzat O, Adu A, Nabhani Gebara S. Telehealth: misconceptions and experiences of healthcare professionals in England. Int J Pharm Pract. 2017.

14 Kassenärztliche Bundesvereinigung, Praxisbarometer Digitalisierung. 2018.

15 Von Gossler C and Klauser C. Telemedizin in der Schweiz - das Beispiel Medgate. Dtsch Medizinische Wochenschrift. 2017. 